\title{
LISA measurement of gravitational wave background anisotropy: Hexadecapole moment via a correlation analysis
}

\author{
Naoki Seto ${ }^{1, *}$ and Asantha Cooray ${ }^{1,2}$ \\ ${ }^{1}$ Theoretical Astrophysics, MC 130-33, California Institute of Technology, Pasadena, California 91125, USA \\ ${ }^{2}$ Department of Physics and Astronomy, University of California, Irvine, California 92687, USA
}

(Received 10 March 2004; published 9 December 2004)

\begin{abstract}
We discuss spatial fluctuations in the gravitational wave background arising from unresolved Galactic binary sources, such as close white dwarf binaries, due to the fact the galactic binary source distribution is anisotropic. We introduce a correlation analysis of the two data streams of the Laser Interferometer Space Antenna (LISA) to extract spherical harmonic coefficients of the hexadecapole moment $(l=4)$ related to the projected two-dimensional density distribution of the binary source population in an independent manner. In this analysis, we use a free parameter with which we can effectively control the frequency of the cartwheel rotation of LISA. The proposed technique complements and improves over previous suggestions in the literature to measure the gravitational wave background anisotropy based on the time modulation of data as LISA orbits around the Sun. Such techniques, however, are restricted only to certain combinations of spherical harmonic coefficients of the galaxy with no ability to separate them individually. With LISA, $m=2,3$ and 4 coefficients of the hexadecapole $(l=4)$ can be measured with signal-to-noise ratios at the level of ten and above in a certain coordinate system. In addition to the hexadecapole coefficients, when combined with the time modulation analysis, the correlation study also can be used, in principle, to measure quadrupole coefficients of the binary distribution.
\end{abstract}

DOI: 10.1103/PhysRevD.70.123005

PACS numbers: 95.55.Ym, 04.80.Nn, 98.62.Sb

\section{INTRODUCTION}

In addition to gravitational waves from massive black holes at cosmological distances, the Laser Interferometer Space Antenna (LISA [1]) is expected to detect the galactic binary source background, such as close white dwarf binaries, with gravitational waves at frequencies between $0.1 \mathrm{mHz}$ to $100 \mathrm{mHz}$ [2]. At frequencies above $3 \mathrm{mHz}$, LISA will resolve a large number of galactic binaries [3], while at the low frequency end, the unresolved population will form a confusion background of gravitational waves. This background is expected to be anisotropic given the fact that galactic binaries trace the density distribution of our galaxy such that the cumulative gravitational wave flux is expected to be concentrated towards the highest density regimes such as the disk and the bulge of the Milky Way. As LISA orbits around the Sun, its response will be sensitive to different regions on the sky and one expects the data stream to modulate as a function of time. This modulation, in return, can be used to extract information related to the density distribution and to reconstruct the anisotropy of the gravitational wave background [4-6]. Note that the binary background can be considered as both a source of noise or a signal. For example, by treating the background as a signal, we can attempt to obtain certain information related to the Galactic structure through anisotropies of the background [4-6]. On the other hand, if the background is treated as a source of noise, we might also discuss how anisotropies

*Electronic address: (seto,asante)@tapir.caltech.edu affect the data analysis and consider certain strategies to minimize any impact [7].

Here, we treat the background as a signal and consider the presence of anisotropies and their potential measurement using a correlation between the two data streams of LISA. For this purpose, following Ref. [7], we introduce a spherical harmonic moment analysis of LISA data in terms of spatial inhomogeneities in the two-dimensional projected density distribution when integrated over the observer to source distance. We also emphasize the subtle use of a freedom related to data combinations that also corresponds to an effective cartwheel rotation angle of LISA detectors in the detector plane. The technique we discuss here complements, and improves, studies based on the time modulation of the data stream and allows one to extract individual coefficients of multipole moments related to the two-dimensional binary source distribution. Prior techniques were restricted only to combinations of coefficients related to the monopole $(l=0)$, quadrupole $(l=2)$, and the hexadecapole $(l=4)$. Considering LISA observational parameters, we show that certain coefficients of the hexadecapole multipole moments can be extracted with signal-to-noise ratios at the level of ten and above.

We also study the complimentary nature of the two methods involving time modulation and the correlation of data streams and suggest that, in combination, one can also, in principle, extract information related to coefficients of the quadrupole distribution. The only multipole moment coefficients that will remain individually undetermined are ones of $l=0, m=0$ mode (monopole), and 
the $l=2, m=0$ mode related to the quadrupole. These two, however, can be determined, in combination, as a sum. The correlation analysis would be one of the crucial elements for LISA's follow-on missions aiming to detect weak gravitational wave background from the early universe [8,9]. While it is expected that the basic design of any potential missions in the long term is likely to be largely different from that of LISA, the correlation analysis with LISA data streams may present an important first step for any planned studies in the future that attempt to use similar procedures to both extract signals or to remove foreground noise.

The discussion is organized as follows: in the next section, we outline the spherical harmonic formulation of the anisotropies in the gravitational wave background and how the low order moments $l=0,2$ and 4 are related to the LISA data streams. In Sec. III, we discuss the correlation between data streams as a way to extract information related to these anisotropies beginning with a brief discussion of prior proposed techniques related to modulations in the data streams. In Sec. IV, we conclude with a summary of our main results. For background information related to the technique presented in this paper, we refer the reader to Ref. [7].

\section{FORMULATION}

In this section, we first discuss the measurement of gravitational wave background with LISA detectors briefly and then discuss how this data is related to the density distribution of the binary background, in terms of the spherical moments of inhomogeneities in the projected distribution on the sky. For the purpose of this discussion, we make use of the signal matrix involving the two data streams.

\section{A. Detector Response}

At the low frequency regime of the LISA band, two separate modes $(A, E)$ in gravitational waves can be independently measured such that the laser frequency noise is reduced to a level below other detector noises. Furthermore, individual detector noises for these two modes do not correlate [10]. We write these two data streams $d_{I}(I=A, E)$, made with detector noise $n_{I}^{D}$, when the response to gravitational waves $h_{I}$ as $d_{I}=h_{I}+$ $n_{I}^{D}$. We define the detector noise spectrum $S_{I J}^{D}$ as $\left\langle n_{I}^{D *}(f) n_{J}^{D}\left(f^{\prime}\right)\right\rangle=\delta\left(f-f^{\prime}\right) S_{I J}^{D}(f) / 2$. The frequency dependence on noise is almost irrelevant for the present discussion and, hereafter, we omit the explicit dependence for notational simplicity. Since the noise in two modes are uncorrelated and have same magnitude due to the symmetric data combinations, we have the following relation for the detector noise spectrum matrix

$$
S_{A A}^{D}=S_{E E}^{D}, \quad S_{A E}^{D}=0 .
$$

The responses $h_{I}$ to the low frequency gravitational waves for two modes $(I=A, E)$ can be essentially regarded as that of two $90^{\circ}$-interferometers rotated by $45^{\circ}$ from each other, as shown in Fig. 1. It is well known that the correlation between two modes like these does not have sensitivity to the monopole $(l=0)$ mode that is primarily important for cosmology. However, as we see later, the correlation is sensitive to the hexadecapole $(l=$ 4) mode of the background. As far as we know, this paper is the first attempt to quantitatively deal with the correlation analysis of the two orthogonal modes of LISA for measurement of the gravitational wave anisotropies. To describe gravitational wave measurements, we take a reference coordinate system $\left(X_{D}, Y_{D}, Z_{D}\right)$ from the detector system, where the $Z_{D}$-axis is normal to the detector plane. The gravitational waves behave as a Spin-2 field such that one can make linear combinations $\left[d_{A}\left(\phi_{f}\right), d_{E}\left(\phi_{f}\right)\right]$ from the original modes $\left[d_{A}, d_{E}\right]$ with a two-dimensional rotation matrix $R(\phi)$ as

$$
\left(\begin{array}{l}
d_{A}\left(\phi_{f}\right) \\
d_{E}\left(\phi_{f}\right)
\end{array}\right)=R\left(2 \phi_{f}\right)\left(\begin{array}{l}
d_{A} \\
d_{E}
\end{array}\right)
$$

These new modes $\left[h_{A}\left(\phi_{f}\right), h_{E}\left(\phi_{f}\right)\right]$ are equivalent to the responses of two $90^{\circ}$-interferometers that are obtained by rotation of the original modes $\left(h_{A}, h_{E}\right)$ with an angle $\phi_{f}$ around the $Z_{D}$-axis (see also [11]). Based on this trans-

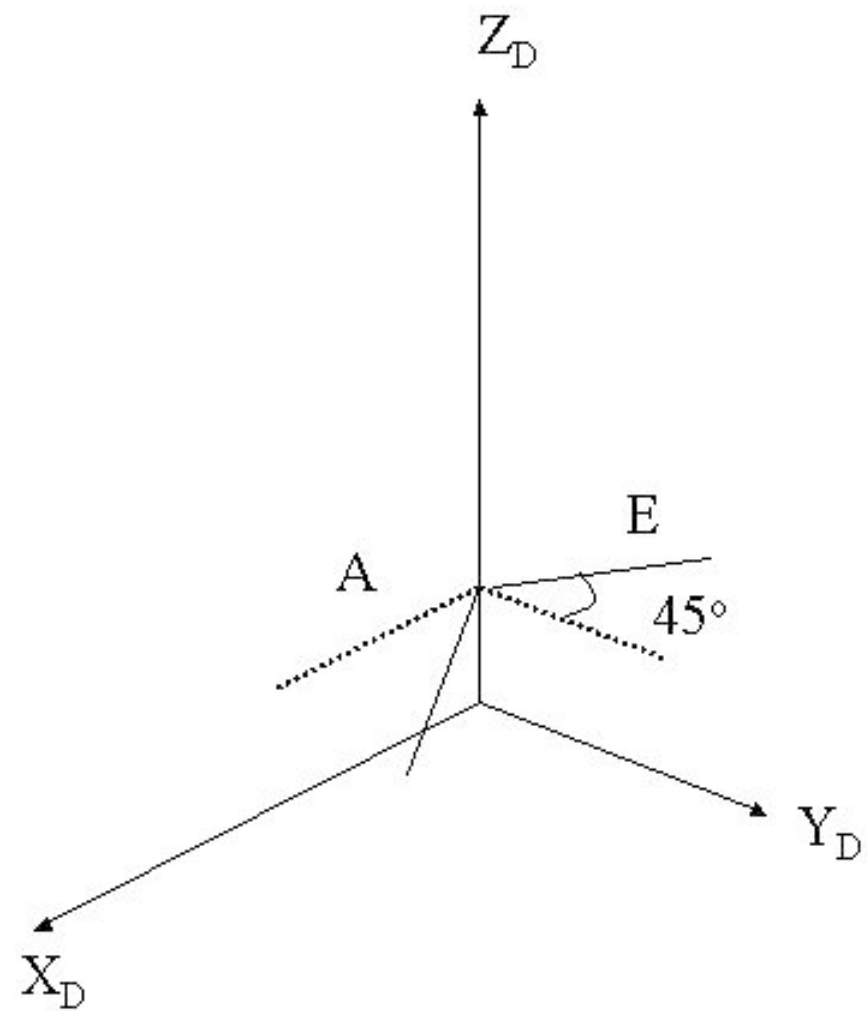

FIG. 1. Definition of the detector coordinate $\left(X_{D}, Y_{D}, Z_{D}\right)$. The $Z_{D}$-axis is normal to the detector plane. A and $\mathrm{E}$ modes can be regarded as two L-shaped detectors rotated by $45^{\circ}$. 
formation, one can easily confirm following relations for the detector noise spectrum for an arbitrary rotational angle

$$
S_{A A}^{D}\left(\phi_{f}\right)=S_{E E}^{D}\left(\phi_{f}\right)=S_{A A}^{D}=S_{E E}^{D}, \quad S_{A E}^{D}\left(\phi_{f}\right)=0 .
$$

Actually, with this degree of freedom, we can effectively control the frequency of the cartwheel rotation of LISA that is fixed at once per year for the original A and E modes. As we discuss later, this freedom (of data combination) is important for the measurement of anisotropies related to the galactic gravitational wave background. This parameter blends the information of the original A and $\mathrm{E}$ modes as their linear combination. Therefore the advantages with using the two modes are closely related to the effects of the parameter.

The signal matrix $S_{I J}^{B}$ for the galactic binary background is defined as in the case of detector noise and is given by the three-dimensional spatial distribution of gravitational wave emitting binaries $\rho$ when integrated over the volume, as

$$
S_{I J}^{B}=P \int d^{3} \boldsymbol{r} \rho(\boldsymbol{r}) r^{-2} F_{I J}(\theta, \phi),
$$

where $P$ is a normalization factor that is not important for our analysis. The weighing factor $r^{-2}$ in the integral is related to the fact that the gravitational wave amplitude scales as $r^{-1}$, where $r$ is the radial distance from the detector. Here, we assume that the frequency distribution and the spatial distribution of galactic gravitational wave emission rate are independent. The functions $F_{I J}$ represent the dependence of the detector's response on the source direction and are defined as

$$
F_{I J}=\frac{1}{2}\left[\left(\frac{1+\cos ^{2} \theta}{2}\right)^{2} p_{1}+(\cos \theta)^{2} p_{2}\right],
$$

with $\left(p_{1}, p_{2}\right)=\left(\cos ^{2} 2 \phi, \sin ^{2} 2 \phi\right)$ for $F_{A A}, \quad\left(p_{1}, p_{2}\right)=$ $\left(\sin ^{2} 2 \phi, \cos ^{2} 2 \phi\right)$ for $F_{E E}$, and $\left(p_{1}, p_{2}\right)=$ $(\sin 2 \phi \cos 2 \phi,-\sin 2 \phi \cos 2 \phi)$ for $F_{A E}$. Using a spherical coordinate we can express Eq. (2.4) as [4]

$$
S_{I J}^{B}=\int d \Omega B(\theta, \phi) F_{I J}(\theta, \phi),
$$

where the angular dependence of the gravitational wave background luminosity $B(\theta, \phi)$ is defined through the radial integral as

$$
B(\theta, \phi) \equiv P \int d r \rho(r, \theta, \phi) .
$$

Note the dependence on $r^{-2}$ factor in Eq. (2.4) is removed due to the $r^{2} d r$ factor involved in the $d^{3} \boldsymbol{r}$ integral. Thus, the projected two-dimensional structure of the density distribution, as seen by gravitational waves, is simply proportional to the column density of the binary population; this also is proportional to the radial distance averaged density profile and, roughly speaking, the function $B(\theta, \phi)$ can be regarded as the optical luminosity distribution on the sky. As before, one can relate the signal matrix under a rotation such that $S_{I J}^{B}\left(\phi_{f}\right)=$ $R\left(2 \phi_{f}\right) S_{I J}^{B} R\left(-2 \phi_{f}\right)$ for the new data combination of $\left[d_{A}\left(\phi_{f}\right), d_{E}\left(\phi_{f}\right)\right]$.

\section{B. Spherical Harmonic Expansion}

As shown in Fig. 1, the noise matrix $S_{I J}^{B}$ is determined by the orientation of the coordinate system $K_{D} ;\left(Z_{D}, Y_{D}, Z_{D}\right)$ which changes with the rotation of LISA. Given this, we consider the spherical harmonic expansion under a fixed coordinate system $K_{0} ;\left(X_{0}, Y_{0}, Z_{0}\right)$ since such a fixed system can aid in analyzing the gravitational wave background $[5,6]$. In this system, we set the $\left(X_{0}, Y_{0}\right)$-plane on the ecliptic and take $X_{0}$-axis in the direction of the autumn equinox from the Sun. This is a reasonable choice considering the LISA configuration [1,7]. The two systems $K_{D}$ and $K_{0}$ are related by the Euler angles $(\alpha, \beta, \gamma)$. Here $(\alpha, \beta)$ is the direction of the $Z_{D}$-axis in the fixed $K_{0}$ system, while $\gamma$ is essentially degenerate with the freedom related to $\phi_{f}$-rotation in Eq. (2.2) around the $Z_{D}$-axis. Therefore, we use the angle $\gamma$ hereafter as an independent variable.

The angular dependence of the gravitational wave intensity $B(\theta, \phi)$ can be decomposed with spherical harmonic moments $Y_{l m}(\theta, \phi)$ (with the angular variables in the $K_{0}$ system) as

$$
B(\theta, \phi)=\sum_{l, m} B_{l m} Y_{l m}(\theta, \phi) .
$$

Here, harmonic coefficients $B_{l m}$ are given as

$$
B_{l m}=\langle\operatorname{lm} \mid B\rangle,
$$

where we have used the traditional notation for the inner product $\langle a \mid b\rangle \equiv \int d \Omega a^{*}(\theta, \phi) b(\theta, \phi)$ and an abbreviation $|l m\rangle \equiv\left|Y_{l m}(\theta, \phi)\right\rangle$. Our goal in this subsection is to write down the matrix $S_{I J}^{B}(\alpha, \beta, \gamma)$, in the moving $K_{D}$ frame that is characterized by the Euler angles $(\alpha, \beta, \gamma)$, with coefficients $B_{l m}$ related to the background density distribution.

First, the matrix $S_{I J}^{B}(\alpha, \beta, \gamma)$ is formally expressed as

$$
S_{I J}^{B}(\alpha, \beta, \gamma)=\left\langle F_{I J}(\theta, \phi)\left|U(\alpha, \beta, \gamma)^{-1}\right| B(\theta, \phi)\right\rangle,
$$

where $U(\alpha, \beta, \gamma)$ is the rotation operator related to Euler angles $\alpha, \beta$, and $\gamma$. After some tedious but straightforward algebra (see, Appendix A of Ref. [7] for details) we write 
TABLE I. Spherical harmonic coefficients of the Galactic binary background. Note that $B_{l-m}=(-1)^{m} B_{l m}^{*}$.

\begin{tabular}{lccccccccc}
\hline \hline$l, m$ & 0,0 & 2,0 & 2,1 & 2,2 & 4,0 & 4,1 & 4,2 & 4,3 & 4,4 \\
\hline$B_{l m} / B_{00}$ & 1 & -0.243 & $-0.292+0.077 i$ & $-0.647-0.038 i$ & 0.052 & $-0.076+0.076 i$ & $0.289-0.012 i$ & $-0.329-0.073 i$ & $0.504+0.061 i$ \\
\hline \hline
\end{tabular}

$$
\begin{aligned}
& S_{A A}^{B}=\frac{2 \sqrt{\pi}}{5} B_{00}+\frac{4 \sqrt{\pi}}{7 \sqrt{5}} \sum_{m} D_{0 m}^{2} \operatorname{Re}\left[e^{i m \alpha} B_{2 m}\right]+\frac{\sqrt{\pi}}{105} \sum_{m} D_{0 m}^{4} \operatorname{Re}\left[e^{i m \alpha} B_{4 m}\right]+\frac{2 \sqrt{\pi}}{3 \sqrt{70}} \sum_{m} D_{4 m}^{4} \operatorname{Re}\left[B_{4 m} e^{(4 \gamma+m \alpha) i}\right], \\
& S_{E E}^{B}=\frac{2 \sqrt{\pi}}{5} B_{00}+\frac{4 \sqrt{\pi}}{7 \sqrt{5}} \sum_{m} D_{0 m}^{2} \operatorname{Re}\left[e^{i m \alpha} B_{2 m}\right]+\frac{\sqrt{\pi}}{105} \sum_{m} D_{0 m}^{4} \operatorname{Re}\left[e^{i m \alpha} B_{4 m}\right]-\frac{2 \sqrt{\pi}}{3 \sqrt{70}} \sum_{m} D_{4 m}^{4} \operatorname{Re}\left[B_{4 m} e^{(4 \gamma+m \alpha) i}\right], \\
& S_{A E}^{B}=\frac{2 \sqrt{\pi}}{3 \sqrt{70}} \sum_{m} D_{4 m}^{4} \operatorname{Im}\left[B_{4 m} e^{(4 \gamma+m \alpha) i}\right] .
\end{aligned}
$$

where the matrix element $D_{s m}^{l}(\beta)$ is defined as $D_{s m}^{l}(\beta)=$ $\left\langle l s\left|U(0, \beta, 0)^{-1}\right| l m\right\rangle$ and given by the Jacobi polynomials $P_{l-s}^{(s+m, s-m)}$. When $s \geq m$, their explicit forms are [12]

$$
\begin{aligned}
D_{s m}^{l}(\beta)= & (-1)^{l-s}\left[\frac{(l-s) !(l+s) !}{(l-m) !(l+m) !}\right]^{1 / 2}\left(\cos \frac{\beta}{2}\right)^{s+m} \\
& \times\left(\sin \frac{\beta}{2}\right)^{s-m} P_{l-s}^{(s+m, s-m)}(-\cos \beta),
\end{aligned}
$$

while for $s<m, D_{s m}^{l}(\beta)=(-1)^{s-m} D_{m s}^{l}(\beta)$. As written in Eq. (2.11), the two data streams from LISA detectors are only sensitive to the background $B_{l m}$ with $l=0,2$, and 4 harmonics. This is simply due to the angular dependence of the $F_{I J}(\theta, \phi)$ term such that $\left\langle F_{I J} \mid l m\right\rangle$ is only nonzero when $l=0,2$, and 4 . Unlike electromagnetic radiation observations - such as that of photons in the cosmic microwave background, where, in principle, all multipole moments of the inhomogeneous radiation field are measurable - with gravitational waves one is only restricted to these low order moments of the underlying field due to the poor directional characterization of the gravitational wave detectors. One can potentially extract additional multipole moments of the gravitational wave background with more data combinations involving, say, an additional configuration such as another set of three spacecrafts $[5,6]$. Since the LISA configuration is fixed to three spacecrafts, we do not discuss such possibilities further. Note that our expressions in Eq. (2.11) are valid at the long wavelength limit of LISA relevant for the Galactic confusion background. At higher frequency regime, we expect the dependence of these terms in terms of harmonic coefficients to change [5,6] (see also [13]).

The off-diagonal element of the noise matrix $S_{A E}^{B}$ is nonzero due to the $l=4$ coefficients of the binary background. The above equations related to $S_{I J}^{B}(\alpha, \beta, \gamma)$ are essentially same as Eqs. (A13)-(A15) in Ref. [7]. Here, in comparison, we have rewritten the spin-weight harmonics in that paper with matrix elements $D_{s m}^{l}(\beta)$ with

$$
-s Y_{l m}(\beta, \alpha) \sqrt{\frac{4 \pi}{2 l+1}}=D_{s m}^{l}(\beta) e^{i m \alpha}
$$

In the case of observations with LISA, the angle $\beta$ is fixed to the value of $\pi / 3$. This leads to the restriction that, in principle, one cannot separate information related to $B_{00}$ and $B_{20}$. The angle $\alpha$ is given as $\alpha=2 \pi t$ with orbital time $t$ in units of year (measured from the autumn equinox). For the galactic binary distribution $\rho(\boldsymbol{r})$ we use the triaxial model given in [14] and used in [7]. The relevant coefficients $B_{l m}$ and $D_{s m}^{l}$ are presented in Tables I and II.

While we primarily discuss the background from our galaxy, it is worth considering the possibility that anisotropies can exist due to gravitational wave emission from binaries in other galaxies of the local group, such as the

\begin{tabular}{|c|c|c|c|c|c|c|c|c|}
\hline & & $D_{0,-2}^{2}$ & $D_{0,-1}^{2}$ & $D_{0,0}^{2}$ & $D_{0,1}^{2}$ & $D_{0,2}^{2}$ & & \\
\hline & & 0.459 & 0.530 & -0.125 & -0.530 & 0.459 & & \\
\hline$D_{0,-4}^{4}$ & $D_{0,-3}^{4}$ & $D_{0,-2}^{4}$ & $D_{0,-1}^{4}$ & $D_{0,0}^{4}$ & $D_{0,1}^{4}$ & $D_{0,2}^{4}$ & $D_{0,3}^{4}$ & $D_{0,4}^{4}$ \\
\hline 0.294 & 0.480 & 0.222 & -0.303 & -0.289 & 0.303 & 0.222 & -0.480 & 0.294 \\
\hline$D_{4,-4}^{4}$ & $D_{4,-3}^{4}$ & $D_{4,-2}^{4}$ & $D_{4,-1}^{4}$ & $D_{4,0}^{4}$ & $D_{4,1}^{4}$ & $D_{4,2}^{4}$ & $D_{4,3}^{4}$ & $D_{4,4}^{4}$ \\
\hline 0.004 & 0.019 & 0.062 & 0.152 & 0.294 & 0.456 & 0.558 & 0.516 & 0.316 \\
\hline
\end{tabular}
Andromeda galaxy (M31) or the Large Magellanic Cloud (LMC). Their contribution to the anisotropy $B(\theta, \phi)$ is roughly proportional to (mass) $/$ (distance) $^{2}$. The characteristic distance is $10 \mathrm{kpc}$ for the Milky Way galaxy,

TABLE II. Matrix elements $D_{s m}^{l}(\beta)$ for $\beta=\pi / 3$. 
$50 \mathrm{kpc}$ for LMC, and $700 \mathrm{kpc}$ for M31. As for the mass, M31 is comparable to Milky Way, but LMC is $\sim 50^{-1}$ times smaller. Given these parameters, we find that the contribution to anisotropies of the gravitational wave background from other members of the local group is negligible. This also is apparent from the fact that the optical luminosity of these and other galaxies are much lower than the Milky Way.

\section{EXTRACTION OF A NISOTROPY PARAMETERS}

Having established the relation between components of the LISA data stream matrix and coefficients of the binary background anisotropy, we now discuss the individual measurement of these anisotropy coefficients based on various combinations of terms in the data matrix. First, we briefly outline the previous method based on the time modulation of the data stream and consider the relation between anisotropy coefficients there and the ones we discuss here.

\section{A. Time Modulation of LISA Data}

Previous studies on the measurement of anisotropies $B_{l m}$ are based on the time modulation of the spectrum $S_{A A}^{B}$ [4-6] (see also [12]). These techniques do not use (i) other modes $S_{A E}^{B}$ or $S_{E E}^{B}$ and (ii) the freedom to adjust one of the Euler angles, $\gamma$, a priori. In time modulation studies, the angle $\gamma$ simply follows the motion of LISA and given as $\gamma=-2 \pi t+\gamma_{0}$ where $\gamma_{0}$ is some constant angle (here, we put $\gamma_{0}=0$ for simplicity). This means that the cartwheel motion of LISA is one rotation per year (see Fig. 2). We follow briefly the underlying idea behind time modulation method and highlight certain problems for the signal extraction related to $B_{l m}$. The function $S_{A A}^{B}$ can be expanded as $S_{A A}^{B}(t)=S_{A A}^{B}(\alpha=2 \pi t, \beta=\pi / 3, \gamma=$ $-2 \pi t)=\sum_{n=-8}^{8} C_{n} e^{2 \pi \text { int }}$. Since $C_{n}^{*}=C_{-n}$, as one is observing a real realization on the sky, we do not discuss $C_{n}$ with $n<0$. The explicit forms of the coefficients $C_{n}$ are easily derived from Eqs. (2.11)

$$
\begin{aligned}
C_{0}= & \frac{2 \sqrt{\pi}}{5} B_{00}+\frac{4}{7} \sqrt{\frac{\pi}{5}} D_{00}^{2} B_{20}+\frac{\sqrt{\pi}}{105} D_{40}^{4} B_{00} \\
& +\frac{2}{3} \sqrt{\frac{\pi}{70}} D_{44}^{4} \operatorname{Re}\left[B_{44}\right] \\
C_{1}= & \frac{4}{7} \sqrt{\frac{\pi}{5}} D_{01}^{2} B_{21}+\frac{\sqrt{\pi}}{105} D_{01}^{4} B_{41}-\frac{1}{3} \sqrt{\frac{\pi}{70}} D_{43}^{4} B_{4-3}, \\
C_{2}= & \frac{4}{7} \sqrt{\frac{\pi}{5}} D_{00}^{2} B_{22}+\frac{\sqrt{\pi}}{105} D_{02}^{4} B_{42}+\frac{1}{3} \sqrt{\frac{\pi}{70}} D_{42}^{4} B_{4-2},
\end{aligned}
$$

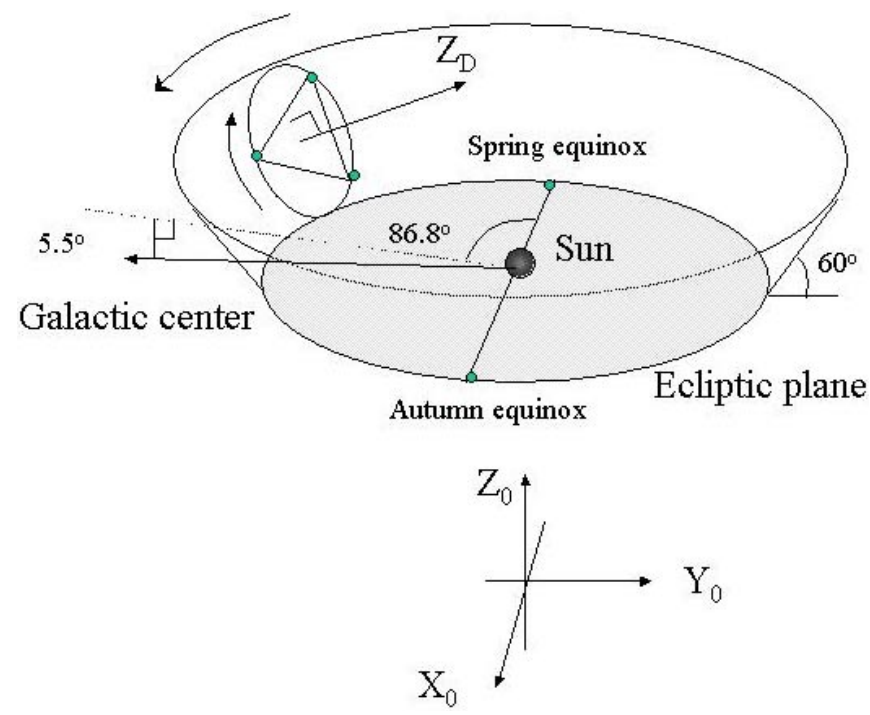

FIG. 2 (color online). Configuration of LISA and the definition of the fixed ecliptic coordinate $\left(X_{0}, Y_{0}, Z_{0}\right)$. The $Z_{0}$-axis is normal to the ecliptic plane and $X_{0}$-axis is oriented to the autumn equinox from the Sun. Three spacecrafts rotate around the Sun and the triangle made by them shows a cartwheel rotation once per year.

$$
\begin{gathered}
C_{3}=\frac{\sqrt{\pi}}{105} D_{03}^{4} B_{43}-\frac{1}{3} \sqrt{\frac{\pi}{70}} D_{41}^{4} B_{4-1}, \\
C_{4}=\frac{\sqrt{\pi}}{105} D_{04}^{4} B_{44}+\frac{1}{3} \sqrt{\frac{\pi}{70}} D_{40}^{4} B_{40}, \\
C_{5}=-\frac{1}{3} \sqrt{\frac{\pi}{70}} D_{4-1}^{4} B_{41}, \quad C_{6}=\frac{1}{3} \sqrt{\frac{\pi}{70}} D_{4-2}^{4} B_{42}, \\
C_{7}=-\frac{1}{3} \sqrt{\frac{\pi}{70}} D_{4-3}^{4} B_{43}, \quad C_{8}=\frac{1}{3} \sqrt{\frac{\pi}{70}} D_{4-4}^{4} B_{44} .
\end{gathered}
$$

Assuming that the detector noise $S_{A A}^{D}$ is significantly smaller than the background $S_{A A}^{B}$ in a frequency bandwidth $\Delta f$, the signal-to-noise ratio for each coefficients $(n \neq 0)$ is given as $[4,6]$

$$
\left(\frac{S}{N}\right)_{n}=\sqrt{2} \frac{\left|C_{n}\right|}{C_{0}} \sqrt{\Delta f T}
$$

where $T$ is the observational period. Statistics for the measurement of the time dependent signal $C_{n}(n \neq 0)$ would be largely different from that of the constant part $C_{0}$. The coefficients $C_{n}$ are complex numbers but their phases depend on the choice of the orientation of the $X_{0}$-axis that is somewhat arbitrary in the ecliptic plane. Therefore, we summed up the signal-to-noise ratios of their real and imaginary parts in Eq. (3.6). The numerical prefactor of $\sqrt{2}$ comes from the fact that we assume the noise contributions to the real and imaginary parts of these coefficients are related to the total noise $N_{T}$ such that $N_{R}=N_{I}=N_{T} / \sqrt{2}$ without correlation between them. For our model parameters related to the binary 
distribution, we have

$$
\begin{array}{lll}
\left(\frac{S}{N}\right)_{1}=50, & \left(\frac{S}{N}\right)_{2}=73, & \left(\frac{S}{N}\right)_{3}=3.6 \\
\left(\frac{S}{N}\right)_{4}=2.2, & \left(\frac{S}{N}\right)_{5}=0.69, & \left(\frac{S}{N}\right)_{6}=0.76 \\
\left(\frac{S}{N}\right)_{7}=0.23, & \left(\frac{S}{N}\right)_{8}=0.084,
\end{array}
$$

with $T=10^{8} \mathrm{~s}$ and $\Delta f=10^{-3} \mathrm{~Hz}$. Thus, one can obtain only $C_{1}$ and $C_{2}$ with sufficient statistical significance. The above signal-to-noise ratios are consistent with previous analyses when considering the difference between models for the Galaxy density distribution between these calculations and the present one [4]. Since $C_{1}$ and $C_{2}$ depend on certain combinations of $B_{l m}$, one cannot separate out the multipole moment coefficients related to the gravitational wave background by simply measuring the time modulation components in the Fourier space. Moreover, in terms where certain $B_{l m}$ coefficients are simply proportional to $C_{n}$ coefficients, especially when $n>5$, the dependence is significantly reduced through small coupling coefficients. For example, the information related to $B_{4 \pm 3}$ appears in $C_{7}$ with the factor $-D_{4-3}^{4} \sqrt{\pi / 70} / 3=-0.0044$. One can consider combinations of $C_{n}$ to extract a certain $B_{l m}$, but, again, for example, for the case with $B_{4 \pm 3}$, the dependence in $C_{3}$ is through $D_{03}^{4} \sqrt{\pi} / 105=-0.0081$ and in $C_{1}$ through $-D_{43}^{4} \sqrt{\pi / 70} / 3=-0.036$. The former factor (in $C_{7}$ ) is smaller than the latter (note $D_{4-3}^{4} \ll D_{40}^{4}, D_{43}^{4}$, Table II), but information related to $B_{43}$ in the latter remains to be degenerate with other $B_{l m}$ coefficients such that one cannot easily find the necessary combinations. The situation is similar also for $B_{4 \pm 2}$ or $B_{4 \pm 4}$. The factor $D_{4-4}^{4} \sqrt{\pi / 70} / 3$ in $C_{8}$ is extremely small, and it is impossible to determine $B_{44}$ form the coefficient $C_{8}$.

From these examples, we learn two things: We should try to improve the signal-to-noise ratio for $B_{l m}$ coefficients by taking a suitable combination with respect to the matrix element $D_{l m}^{4}$ and handle the degeneracy of $B_{l m}$ coefficients with the use of other modes.

In the next subsection, we study a method for signal analysis where we pay special attention to these two points.

\section{B. Correlation Analysis}

We use the correlation analysis $\int S_{A E}(t) d t$ to measure the parameter $B_{4 m}$. By setting $\gamma=-\pi m^{\prime} t / 2+\pi / 8$ $\left(m^{\prime} \geq 0\right)$, for each $m^{\prime}$ from 0 to 4 , a priori-since we have this freedom to specify this angle beforehand-one obtains, under the scenario that $\alpha=2 \pi t$ and after integrating the signal $S_{A E}(t)$ over an observational period $T$ (an integer in units of $1 \mathrm{yr}$ ),

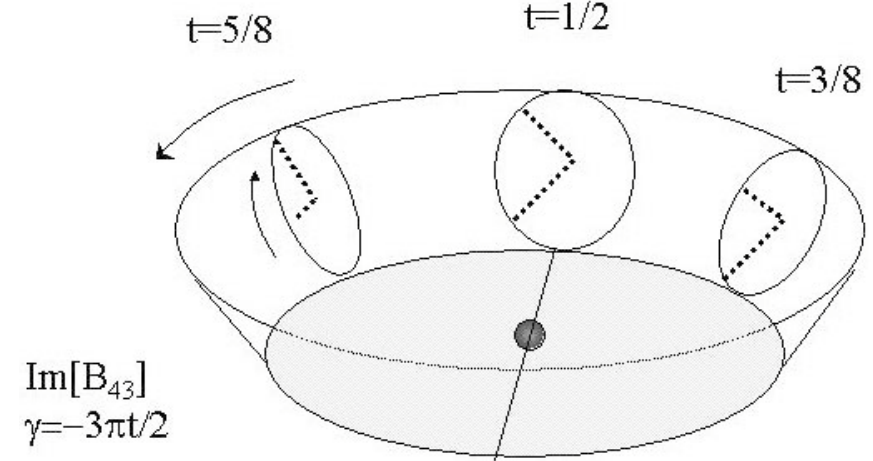

FIG. 3. Rotation of the effective $90^{\circ}$ detector A for the parameter $\gamma=-3 \pi t / 2$. We show the situation around the spring equinox $(t=1 / 2)$. This $\gamma$ is the optimized choice for measurement of $\operatorname{Im}\left[B_{43}\right]$ with the time integral Eq. (3.8).

$$
\begin{aligned}
\left\langle S_{A E}^{B}\right\rangle_{R}^{m^{\prime}} & \equiv \int S_{A E}^{B}(t) d t \\
& =\int d t \frac{2 \sqrt{\pi}}{3 \sqrt{70}} \sum_{m} D_{4 m}^{4} \operatorname{Im}\left[B_{4 m} e^{i\left[2 \pi\left(m-m^{\prime}\right) t+\pi / 2\right]}\right] \\
& =-\frac{2 \sqrt{\pi}}{3 \sqrt{70}} D_{4 m^{\prime}}^{4} \operatorname{Re}\left[B_{4 m^{\prime}}\right],
\end{aligned}
$$

while the imaginary component of the same quantity can be obtained by setting $\gamma=-\pi / 2 m^{\prime} t$ such that $\left\langle S_{A E}^{B}\right\rangle_{I}^{m} \propto$ $\operatorname{Im}\left[B_{4 m}\right]$. Thus, the substitution for $\gamma$ has the advantage that one can now individually get real and imaginary parts of $B_{4 m}$ while at the same time also making use of the substantially large coupling coefficients of $D_{4 m}^{4}(m \geq$ $0)$ that are associated with harmonic coefficients. In Fig. 3 we show how the effective detector $A$ rotates within the detector plane with a choice $\gamma=-3 \pi t / 4$ for measurement of $\operatorname{Im}\left[B_{43}\right]$. The frequency of the cartwheel rotation is $2 / 3$ per year. In Fig. 4 we also show the choice $\gamma=$ $-\pi / 8$ for $\operatorname{Re}\left[B_{40}\right]=B_{43}$. The cartwheel rotation is stopped for the latter.

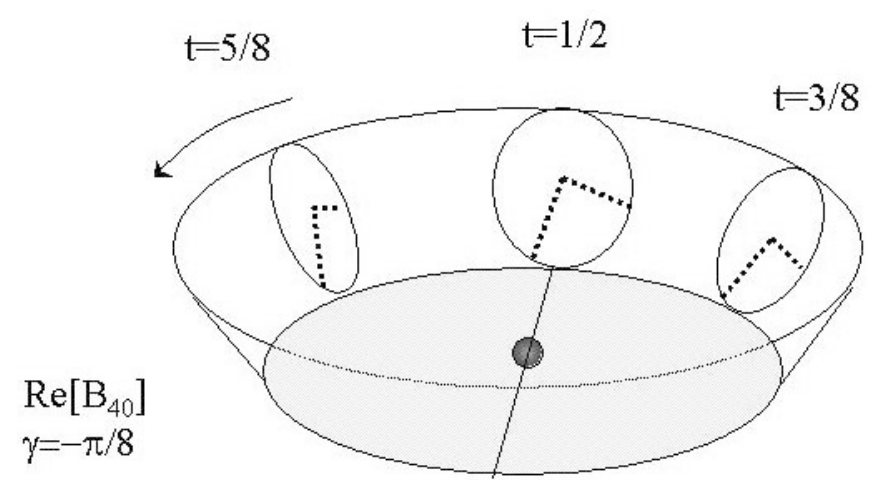

FIG. 4. The case with the parameter $\gamma=-\pi / 8$. This $\gamma$ is a optimized choice for measurement of $\operatorname{Re}\left[B_{43}\right]$ and the cartwheel rotation is now stopped. 
When we neglect the detector noise and the frequency dependence of anisotropies, which would be tolerable for the present study, the signal-to-noise ratio of the correlation analysis, for an individual $l=4$ and $m$ coefficient, is

$$
(\mathrm{S} / \mathrm{N})_{4 m}=\left(\int d t \int d f \frac{\left[\left\langle S_{A E}^{B}\right\rangle^{m}\right]^{2}}{S_{A A}(t) S_{E E}(t)}\right)^{1 / 2},
$$

where we have now combined the real and imaginary parts $\left[\left\langle S_{A E}^{B}\right\rangle^{m}\right]^{2}=\left[\left\langle S_{A E}^{B}\right\rangle_{R}^{m}\right]^{2}+\left[\left\langle S_{A E}^{B}\right\rangle_{I}^{m}\right]^{2}$. We have numerically calculated these signal-to-noise ratios for each $m$ value using the same density distribution model for the galactic binaries as before and we obtain following values for $(\mathrm{S} / \mathrm{N})_{4 m}$ for the measurement of $B_{4 m}$ coefficients, separately, as

$$
\begin{aligned}
& (\mathrm{S} / \mathrm{N})_{40}=1.1, \quad(\mathrm{~S} / \mathrm{N})_{41}=3.7, \quad(\mathrm{~S} / \mathrm{N})_{42}=12 \\
& (\mathrm{~S} / \mathrm{N})_{43}=13,
\end{aligned}
$$

when $\Delta f=10^{-3} \mathrm{~Hz}$ and $T=10^{8} \mathrm{~s}$. Thus, as estimated, we expect one can estimate the coefficients $B_{42}, B_{43}$, and $B_{44}$ accurately, while upper limits can be established for $B_{40}$ and $B_{41}$. Then, using the previous time modulation method, one can further estimate $B_{21}$ from $C_{1}$ coefficient and $B_{22}$ from $C_{2}$ coefficient. Since coefficients $C_{1}$ and $C_{2}$ are dominated by $B_{12}$ and $B_{22}$, respectively, this determination can be carried out with sufficient signal-to-noise ratio.

So far, we have studied the correlation analysis for estimating $B_{4 m}$ individually for all $m$ values and the combination of time modulation related to $S_{A A}^{B}$ and the correlation analysis to extract certain coefficients related to the quadrupole. One can also use the time modulation method alone but with combinations of $S_{A A}^{B}, S_{E E}^{B}$, and $S_{A E}^{B}$. Again, the freedom related to the angle $\gamma$ is useful. An example is the modulation of $S_{A A}^{B}-S_{E E}^{B}$. For example by setting $\gamma=2 \pi t(-m / 4+2)$ or $2 \pi t(-m / 4+2)+\pi / 8$, we can estimate the real and imaginary parts of $B_{4 m}$ from the coefficient $C_{8}$ related to the combination $S_{A A}^{B}-$ $S_{E E}^{B}$. By making numerical estimates, we find that the signal-to-noise ratio for such a measurement is similar to the one estimated above with the correlation analysis. As we commented earlier, this parameter blends the information of the fixed original $\mathrm{A}$ and $\mathrm{E}$ modes that has all the information we want. In contrast to Sec. III A, we can easily reconstruct them from a single data $S_{A A}^{B}$ with fully using the parameter $\gamma$ and then deconvolve the relevant coefficients.

While the combination with time modulation for $S_{A A}^{B}$ (without the freedom of the parameter $\gamma$ ) alone do lead to certain coefficients of $B_{4 m}$, we find that the correlation analysis improves the previous time modulation method by providing a mechanism to extract all coefficients. Moreover, the correlation method complements techniques related to the time modulation and we find that, in combination, the methods can be used to extract most coefficients related to the $l=2$ and 4 moments of the galactic distribution of binaries. The only coefficients that are not independently measured are $B_{00}$ and $B_{20}$, though the two can be measured in combination as a sum. As mentioned earlier, signal analysis for the $C_{0}$ mode is different from other $C_{n}$ modes with $n \neq 0$. For this purpose, data streams other than $A$ and $E$ modes, e.g., Sagnac, would be important to calibrate the detector noise [15].

\section{SUMMARY}

In this paper, we have discussed spatial fluctuations in the gravitational wave background arising from unresolved galactic binary sources, such as close white dwarf binaries, due to the fact the this galactic source distribution, when projected on the sky, is anisotropic. We introduce a spherical harmonic analysis of this anisotropy based on a correlation study of the two data streams of LISA, and propose the measurement of individual spherical harmonic coefficients related to the hexadecapole moment $(l=4)$ in addition to coefficients of the quadrupole $(l=2)$.

The proposed technique complements and improves over previous suggestions in the literature to measure the gravitational wave background anisotropy based on the time modulation of the single data stream [e.g., $\left.S_{A A}^{B}(t)\right]$ as LISA orbits around the Sun and is restricted only to combinations of spherical harmonic coefficients with no ability to separate them. The correlation analysis, by making use of a single freedom related to the rotation angle of detectors in the detector plane or similarly how data are combined, provides a method to independently extract multipole coefficients of the hexadecapole moment of the background. The information related to the quadrupole comes from the correlation analysis in combination with the time modulation. The only independently undetermined coefficients turn out to be that of the monopole and the $m=0$ mode of the quadrupole, though the two can be determined as a sum.

With LISA, we have shown that certain coefficients of the hexadecapole can be measured with signal-to-noise ratios at the level of ten and above. While LISA will present a first step towards a correlation analysis of the gravitational wave background, future missions are likely to exploit this in detail both as a source of signal, to extract spatial information, and noise, to decrease noise to detect almost isotropic backgrounds such as the one related to primordial gravitational waves. This would certainly be the case at low frequencies as the foreground is likely to be the dominant source of anisotropy. At few $\mathrm{mHz}$ frequencies, the fluctuation amplitude related to the extragalactic component is expected to be at the level of a few percent but restricted to arcminute angular scales where galaxy density field is observed to vary. At $\mu \mathrm{Hz}$ frequencies, where the merging massive black holes are 
likely to be the dominant foreground source, the anisotropy will be dominated by the shot-noise related to the finite number density of such binaries on the sky. On the other hand, the anisotropy of the primordial gravitational wave background is expected to have a pattern close to that of the cosmic microwave background, with a fluctuation level of $\delta \sim 10^{-5}$, at large angular scales. A confirmation of their similarity in anisotropy can be used as a proof on the primordial nature of the background. For a direct detection of spatial fluctuations in the gravitational wave background, in the optimistic case where the detector noise is dominated by the monopole mode of the background, one is forced to gravitational wave frequencies at the level of $f \sim \Delta f \sim 10^{4}(\mathrm{SN} / 10)^{2} \times$ $\left(\delta / 10^{-5}\right)^{-2}(T / 3 \mathrm{yr}) \mathrm{Hz}$. Thus, for a detection of the an- isotropy at a level $\delta \sim 10^{-5}$ over a realistic observational period with significant signal-to-noise ratio, the relevant frequencies are at $f \gtrsim 10 \mathrm{kHz}$. Interestingly, at this high end of the gravitational wave spectrum, there are no viable astrophysical sources to suggest that, in the future, one may in fact be able to extract anisotropy associated with the primordial gravitational wave background.

\section{ACKNOWLEDGMENTS}

This work was supported in part by DOE DE-FG0392-ER40701, the Sherman Fairchild foundation (AC), NASA Grant Nos. NAG5-10707, NNG04GK98G, and the Japan Society for the Promotion of Science.
[1] P. L. Bender et al., "LISA Pre-Phase A Report," Second edition, (1998).

[2] R. F. Webbink and Z. Han, in Laser Interferometer Space Antenna, Second International LISA Symposium on the Detection and Observation of Gravitational Waves in Space, AIP Conf. Proc. No. 456 (AIP, New York, 1998) p. 61; G. Nelemans, L. R. Yungelson, and S. F. Portegies Zwart Astron. Astrophys. 375, 890 (2001); N. Seto, Mon. Not. R. Astron. Soc. 333, 469 (2002); A. Cooray, A. J. Farmer, and N. Seto, Astrophys. J. 601, L47 (2004).

[3] G. Nelemans, L. R. Yungelson, S. F. Portegies Zwart, and F. Verbunt, Astron. Astrophys. 365, 492 (2001); N. J. Cornish and S. L. Larson, Phys. Rev. D 67, 103001 (2003); D. Hils, P. L. Bender, and R. F. Webbink, Astrophys. J. 360, 75 (1990).

[4] G. Giampieri and A. G. Polnarev, Mon. Not. R. Astron. Soc. 291, 149 (1997); G. Giampieri and A. G. Polnarev, Classical Quantum Gravity 14, 1521 (1997).

[5] N. J. Cornish, Classical Quantum Gravity 18, 4277 (2001).
[6] C. Ungarelli and A. Vecchio, Phys. Rev. D 64, 121501 (2001).

[7] N. Seto, Phys. Rev. D 69, 123005 (2004).

[8] C. Ungarelli and A. Vecchio, Phys. Rev. D 63, 064030 (2001); N. Seto, S. Kawamura, and T. Nakamura, Phys. Rev. Lett. 87, 221103 (2001).

[9] B. Allen, gr-qc/9604033; M. Maggiore, Phys. Rep. 331, 283 (2000).

[10] T. A. Prince, M. Tinto, S. L. Larson, and J.W. Armstrong, Phys. Rev. D 66, 122002 (2002).

[11] K. R. Nayak, S. V. Dhurandhar, A. Pai, and J. Y. Vinet, Phys. Rev. D 68, 122001 (2003).

[12] B. Allen and A. C. Ottewill, Phys. Rev. D 56, 545 (1997).

[13] H. Kudoh and A. Taruya, gr-qc/0411017.

[14] J. Binney, O. Gerhard, and D. Spergel, Mon. Not. R. Astron. Soc. 288, 365 (1997).

[15] M. Tinto, J.W. Armstrong, and F. B. Estabrook, Phys. Rev. D 63, 021101 (2001); C. J. Hogan and P. L. Bender, Phys. Rev. D 64, 062002 (2001). 